\title{
First Trimester Alcohol Exposure Alters Placental Perfusion and Fetal Oxygen Availability Affecting Fetal Growth and Development in a Non-human Primate Model
}

\author{
Jamie O. Lo, $\mathrm{MD}^{1}$, Matthias C. Schabel, $\mathrm{PhD}^{2}$, Victoria H.J. Roberts, $\mathrm{PhD}^{3}$, Xiaojie Wang,

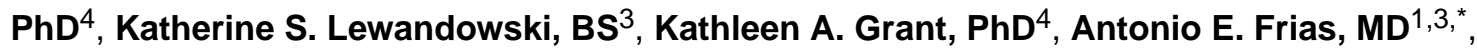 \\ and Christopher D. Kroenke, PhD $^{2,4, *}$ \\ ${ }^{1}$ Department of Obstetrics and Gynecology, Oregon Health \& Science University, Portland, OR \\ ${ }^{2}$ Advanced Imaging Research Center, Oregon Health \& Science University, Portland, OR \\ ${ }^{3}$ Division of Reproductive \& Developmental Sciences, Oregon National Primate Research Center, \\ Oregon Health \& Science University, Beaverton, OR \\ ${ }^{4}$ Division of Neuroscience, Oregon National Primate Research Center, Oregon Health \& Science \\ University, Beaverton, OR
}

\section{Abstract}

Objective-Prenatal alcohol exposure leads to impaired fetal growth, brain development, and stillbirth. Placental impairment likely contributes to these adverse outcomes, but the mechanisms and specific vasoactive effects of alcohol linking altered placental function to impaired fetal development remain areas of active research. Recently, we developed MRI techniques in nonhuman primates to characterize placental blood oxygenation through measurements of $T_{2}{ }^{*}$, and perfusion using dynamic contrast-enhanced (DCE) MRI. The objective of this study was to evaluate the effects of first trimester alcohol exposure on macaque placental function and to characterize fetal brain development, in vivo.

Study Design-Timed-pregnant Rhesus macaques $(\mathrm{n}=12)$ were divided into 2 groups: control $(n=6)$ and ethanol exposed $(n=6)$. Animals were trained to orally self-administer either $1.5 \mathrm{~g} / \mathrm{kg} /$ day of a $4 \%$ ethanol solution (equivalent to 6 drinks/day) or an isocaloric control fluid from pre-conception until gestational day 60 (G60, term is G168). All underwent Doppler ultrasound (D-US) followed by MRI consisting of $T_{2}^{*}$ and DCE measurements. D-US was used to measure uterine artery (Uta) and umbilical vein velocimetry and diameter to calculate Uta volume

Corresponding Author: Jamie Lo, MD, Department of Obstetrics and Gynecology, Oregon Health \& Sciences University, 3181 SW Sam Jackson Park Road, Mail Code L458, Portland, Oregon 97239, Work Phone: (503) 494-2101, Home Phone: (503) 679-2025, Fax: (503) 494-5296, loj@ohsu.edu.

These authors contributed equally to this work

Publisher's Disclaimer: This is a PDF file of an unedited manuscript that has been accepted for publication. As a service to our customers we are providing this early version of the manuscript. The manuscript will undergo copyediting, typesetting, and review of the resulting proof before it is published in its final citable form. Please note that during the production process errors may be discovered which could affect the content, and all legal disclaimers that apply to the journal pertain.

The authors report no conflicts of interest.

Presented orally at the $37^{\text {th }}$ Society of Maternal Fetal Medicine Annual Meetings in Las Vegas, Nevada, January 23-28 ${ }^{\text {th }}, 2017$

Reprints will not be available. 
blood flow (cQuta) and placental volume blood flow (cQuv). After non-invasive imaging, animals underwent C-section delivery for placenta collection and fetal necropsy at G110 (n=6) or G135 $(n=6)$.

Results-Fetal weight and biparietal diameter were significantly smaller in ethanol exposed compared to controls at G110. By D-US, cQuta was decreased ( $\mathrm{p}=0.1)$ and cQuv was significantly lower ( $\mathrm{p}=0.04)$ at both $\mathrm{G} 110$ and G135 in ethanol exposed versus control animals. A significant reduction in placental blood flow was evident by DCE-MRI. As we demonstrated recently, $T_{2}{ }^{*}$ values vary throughout the placenta, and reveal gradients in blood deoxyhemoglobin concentration ranging from highly oxygenated blood (long $T_{2}^{*}$ ) proximal to spiral arteries to highly deoxygenated blood (short $T_{2}{ }^{*}$ ). Distributions of $\mathrm{T}_{2} *$ throughout the placenta show significant global reduction in $T_{2}^{*}$ (and hence high blood deoxyhemoglobin concentration) in ethanol exposed vs. control at $\mathrm{G} 110$ ( $\mathrm{p}=0.02$ ). Fetal brain measurements indicated impaired growth and development at G110, but less so at G135 in ethanol exposed vs. control.

Conclusion-Chronic first trimester ethanol exposure significantly reduces placental perfusion and oxygen supply to the fetal vasculature later in pregnancy. These perturbations of placental function are associated with fetal growth impairments. However, differences between ethanolexposed and control animals in placental function and fetal developmental outcomes were smaller at G135 than at G110. These findings are consistent with placental adaptation to early perturbations allowing for compensated placental function and maintenance of fetal growth.

\section{Keywords}

Alcohol; non-human primate; non-invasive imaging; placental perfusion; fetal oxygenation; fetal growth restriction

\section{INTRODUCTION}

In the United States, approximately $40 \%$ of women consume alcohol in pregnancy ${ }^{1}$ and more than three million are at risk of exposing their fetus to alcohol since more than half of all pregnancies are unplanned and most women do not recognize they are pregnant until four to six weeks post conception ${ }^{2-4}$. Of significance, ethanol readily crosses the placenta and accumulates in the fetus at concentrations proportionate to maternal blood levels within an hour ${ }^{5}$. Prior pregnant ovine ${ }^{6}$, baboon ${ }^{7}$, and perfused human placenta ${ }^{8}$ models have shown that uterine blood flow decreases following acute exposure to ethanol ${ }^{6,7,9}$. In addition, other studies have demonstrated that prenatal ethanol exposure negatively affects fetal growth ${ }^{10}$ and increases the risk of stillbirth, fatty hepatic degeneration and fetal alcohol syndrome ${ }^{11}$. However, the mechanisms and specific vasoactive effects of alcohol exposure early in gestation linking placental perfusion and oxygenation to impaired fetal development are not known.

Alcohol exposure has been shown in vitro to produce dose-dependent placental vasoconstriction that increases fetal-placental vascular resistance and placental perfusion pressure resulting in impaired oxygen transport ${ }^{9}$. The existing literature is limited and consists mostly of in vitro studies of the placenta. Furthermore, human studies are limited by current imaging capabilities to assess placental function in vivo in human subjects ${ }^{12}$. 
Traditionally, Doppler ultrasound (Doppler-US) has been used to query major maternal vessels supporting the placenta for clinical antenatal surveillance. However, this method cannot be used to assess blood flow within the placental intervillous space, the site of maternal-fetal oxygen and nutrient exchange. Recent work with pregnant nonhuman primates (NHP) has identified MRI based methods to characterize maternal blood flow and oxygen exchange in the placenta with the fetal vasculature ${ }^{13}, 14$. Maternal perfusion of the placenta can be quantified using dynamic contrast-enhanced MRI (DCE-MRI), which requires intravenous administration of an MRI contrast reagent ${ }^{13}$. Although we have shown that maternal gadolinium (Prohance ${ }^{\circledR}$ ) administration in rhesus macaques results in minimal fetal exposure ${ }^{15}$, methods for antenatal in vivo hemodynamic assessment not requiring gadolinium-based contrast reagents are clinically desirable. Another method, which involves the analysis of endogenous MRI contrast provided by water $T_{2}^{*}$ values, can quantify maternal blood oxygenation through the blood oxygen level-dependent (BOLD) effect ${ }^{16}$. This provides a safe alternative to standard MRI contrast agents for future clinical use in human subjects.

The NHP model provides a powerful translational model for human pregnancy studies; NHP have a gestational term and developmental ontogeny similar to humans, including its placental structure and function ${ }^{17,18}$. Further benefits to studying NHP subjects are the similar rates of absorption and metabolism of ethanol to humans ${ }^{19}$ and the ability to induce oral self-administration of pre-set doses of ethanol. Maternal smoking, nutritional inadequacy, medication or illicit drug use, all factors that can have a synergistic effect on alcohol exposure, often confound human studies of placental impairment. Modeling human drinking behavior with NHP models provides the added advantage of a precise alcohol history ${ }^{20,21}$ while retaining the normal route of alcohol administration as opposed to strategies used to study fetal ethanol exposure in rodents. Further, voluntary ethanol selfadministration also obviates the need for procedures such as gavage, which introduce confounds associated with increased maternal stress ${ }^{22}$. This study focuses on the effects of chronic early alcohol exposure on placental perfusion and fetal oxygen availability and development in a relevant translational model. We hypothesized that alcohol exposure early in pregnancy would impair maternal perfusion of the placenta resulting in decreased tissue oxygenation.

\section{MATERIALS AND METHODS}

\section{Experimental Design}

A cohort of time-mated pregnant control Rhesus macaques ( $\mathrm{n}=12)$ were divided into 2 groups: control $(\mathrm{n}=6)$ and ethanol exposed $(\mathrm{n}=6)$. Dams were trained to orally selfadminister either $1.5 \mathrm{~g} / \mathrm{kg} /$ day of $4 \%$ ethanol solution (equivalent to 6 drinks/day) or an isocaloric control fluid following a previously described 4-month schedule induced polydipsia procedure ${ }^{20,21,23}$. Following drinking induction, plasma estradiol and progesterone levels were sampled daily until ovulation was suspected, at which point dams were paired with male animals. Plasma estradiol was determined daily and once a decline from peak value was observed, the pairing phase was concluded. The day of peak plasma estradiol was defined to be gestational day 0 (G0). Each pregnant animal continued drinking 
$1.5 \mathrm{~g} / \mathrm{kg} /$ day ethanol until G60 (term is G168), at which point access to ethanol, or isocaloric solution, was removed. A weekly maternal weight was collected for all animals throughout pregnancy. All animals completed an unsedated prenatal ultrasound at G50 to measure the fetal biparietal diameter. Subsequently, all animals underwent Doppler-US followed by MRI consisting of $T_{2}^{*}$ and DCE measurements. After non-invasive imaging, on the same day, animals underwent immediate cesarean section delivery for placenta collection and fetal necropsy at G110 (n=6) or G135 (n=6). Placental and fetal brain tissue was collected, weighed and measured post-delivery.

\section{Imaging}

Doppler-US-All G50 prenatal ultrasounds were performed on awake animals by a single sonographer (J.O.L). Prior to terminal Doppler-US, animals were sedated by intramuscular administration of $10 \mathrm{mg} / \mathrm{kg}$ ketamine (Henry Schein Animal Health ${ }^{\circledR}$ ). Sedation was maintained on a portable anesthesia delivery system providing $\mathrm{O}_{2}$ with $1.5 \%$ isoflurane. Doppler-US measurements were collected by a sonographer (J.O.L) using image-directed pulsed and color Doppler equipment (GE Voluson 730) with a 5- to 9-MHz sector probe. The lowest high-pass filter level was used $(100 \mathrm{~Hz})$, and an angle of $15^{\circ}$ or less between the vessel and the Doppler beam was deemed acceptable. Blood flow velocity waveforms were obtained from the proximal portion of the uterine artery (Uta) as previously described ${ }^{24-26}$. Doppler waveform measurements for the Uta and umbilical artery were performed and averaged over three cycles using machine-specific software and the following measurements were obtained: pulsatility index (PI), velocity time integral (VTI), and fetal heart rate $(\mathrm{HR})^{24-26}$. The diameter of the Uta was measured using power angiography as previously described $^{24-26}$. The cross sectional area (CSA) of the vessel was calculated as CSA $=$ $\pi(\text { diameter } / 2)^{2}$. Uterine artery volume blood flow (cQuta $)$ was calculated using the following formula and corrected by maternal weight: $\mathrm{cQ}_{\mathrm{Uta}}=\mathrm{VTI} \times \mathrm{CSA} \times \mathrm{HR}$. For the placental volume blood flow (cQUV), the Doppler waveforms were obtained from the straight portion of the intra-abdominal umbilical vein (UV) as previously described ${ }^{24-27}$. The mean velocity $\left(\mathrm{V}_{\text {mean }}\right)$ was calculated as 0.5 of the maximum velocity. cQuV was calculated as: $\mathrm{V}_{\text {mean }} \times \mathrm{CSA} \times 60$.

MRI-Immediately following the ultrasound procedures, MRI studies were performed on a nonhuman primate-dedicated 3T Siemens TIM-Trio scanner (Erlangen, Germany) using a circularly-polarized (CP) transmit, 15-channel receive radiofrequency (RF) "extremity" coil (QED, Cleveland, $\mathrm{OH}$ ) to acquire $T_{2}$-weighted images of the fetal brain as described previously ${ }^{28}$. For the analyses of the cerebral cortex, surface models of the interface between CSF and cortical gray matter (the pial cortical surface) was generated using the CARET software package (http://brainvis.wustl.edu). Surface area of each hemisphere was measured using standard functionalities of the CARET program. Placenta MRI followed previously described procedures ${ }^{14,29}$. Anatomic and multiecho imaging was performed during expiratory breath holding, achieved by temporarily suspending ventilation, while DCE-MRI data were acquired during ventilated breathing. Physiological monitoring of pulse rate, arterial blood oxygen saturation, and end-tidal $\mathrm{CO}_{2}$ partial pressure was performed throughout the imaging study, with no deviations from normal ranges observed in these parameters. Spiral artery flow from DCE-MRI data was measured as described previously in 
Frias et $\mathrm{al}^{13}$, and the flow permeability ratio, $F(v P S)$, was determined as described in Schabel et al ${ }^{14}$.

\section{Statistical Analysis}

Data are expressed as mean \pm SD. All animals $(n=12)$ were analyzed and differences between ethanol exposed and controls at G110 and G135 were tested by a non-paired t-test. The $T_{2} *$ results were evaluated using a two-sample Kolmogorov-Smirnov test.

\section{RESULTS}

\section{Growth parameters}

At G50, the fetal biparietal diameter on ultrasound of all the animals in both treatment groups was appropriate for gestational age ${ }^{30}$ measuring on average $1.24 \pm 0.02 \mathrm{~cm}$. Subsequent ultrasounds performed at G110 were notable for a significantly smaller fetal biparietal diameter $(\mathrm{p}<0.05)$ and femur length $(\mathrm{p}<0.01)$ in ethanol exposed animals compared with controls (Table1). Such differences were not observed at G135. Biometry results were reflected in the fetal birth weight; ethanol exposed fetuses weighed significantly less than control animals at both G110 and G135 (p<0.05) (Table 2). Maternal and placental weights were not significantly different across treatment groups (Table 2). Fetal gender ratio was also not significantly different (Table 2).

\section{Placental perfusion and oxygenation}

By Doppler-US, we demonstrated a significant reduction in cQuv in the ethanol exposed group compared to controls at G110 that was not observed at G135 (Table 3). There was no significant difference in cQuta as well as uterine artery and umbilical artery pulsatility indices in either treatment group at both gestational ages (Table 3). Maternal perfusion of the placental intervillous space was evaluated using DCE-MRI as well as $T_{2}^{*}$ measurements, and this revealed differences between ethanol exposed animals and controls. Through the analysis of DCE-MRI measurements, it is possible to identify individual spiral arteries, and quantify maternal volume blood flow, $F$, into the placenta through each of them ${ }^{13}$. The total volumetric blood flow to all placental cotyledons was smaller in the ethanol exposed group versus controls (Table 3), consistent with the semi-quantitative measures of the cQuta, in animals evaluated at G110, but not G135. Figure 1 shows images that reflect perfusion for a representative ethanol exposed case ( $1 \mathrm{a}$ and $1 \mathrm{~b}$ ) and for a representative control case (1c and 1d). Further, through the analysis of water $T_{2}{ }^{*}$ values throughout the placenta, it is possible to quantify the flow-permeability ratio, $F(v P S)$, which relates to spiral artery flow and oxygen delivery to the fetal blood (through the volume fraction of the intervillous space, $v$, and the oxygen permeability surface area product, $P S$ ) on an individual spiral artery basis ${ }^{14}$. In control placentas at both G110 and G135, MR image voxels proximal to spiral artery sources of oxygenated maternal blood are characterized by relatively long $T_{2}^{*}$ values, as previously reported ${ }^{14}$. At greater distances from the spiral arteries, the concentration of deoxyhemoglobin is higher, due to fetal oxygen uptake, resulting in shorter $T_{2}^{*}$ values. The histograms shown in Figure 2 summarize placental $T_{2}{ }^{*}$, demonstrating a statistically significant ( $\mathrm{p}=0.02$ ) reduction in values at G110 in the ethanol exposed cases (red trace) compared to control animals (black traces). Similarly to the DCE-MRI data, the difference 
in $T_{2}^{*}$ observed at G135 between was not statistically significant ( $\left.\mathrm{p}=0.39\right)$. Median $F /(v P S)$ ratios are smaller in ethanol exposed animals than in controls at both G110 and G135, however due the influence of additional factors in the analysis of $T_{2}^{*}$ that contribute to interanimal variation in $F /(v P S)$, such as the number of spiral arteries ${ }^{14}$, significant differences in $F(v P S)$ were not observed (Table 3$)$.

To illustrate the concordance between DCE-MRI and $T_{2}^{*}$ measurements, placental blood flow obtained by DCE-MRI is superimposed on maps of $T_{2}^{*}$ values for both control (Figure 3a-c) and ethanol exposed (Figure 3d-f) animals. As is apparent in Figure 3, a larger number of spiral arteries was noted on DCE-MRI and uniformly higher $T_{2}^{*}$ values were observed in control animals relative to those in the ethanol exposed group.

\section{Fetal brain development}

Fetal brain weights were significantly lower in animals exposed to ethanol (p-0.04) than controls at G110, but not at G135 (Table 4). $T_{2}$-weighted images of the fetal brain were acquired $^{28}$ to measure brain volume and surface area. These revealed the smaller brain volumes measured in ethanol exposed cases compared with controls at G110 $(\mathrm{p}=0.1)$ and G135 ( $\mathrm{p}=0.3$ ) respectively (Table 4$)$. The $T_{2}$-weighted images were used to generate models of the cerebral cortical surfaces and the surface area of the isocortex was reduced $(\mathrm{p}=0.14)$ in ethanol exposed animals versus controls at both gestational time points (Table 4). The above factors suggest that cerebral growth was perturbed by early chronic alcohol exposure.

\section{COMMENT}

In this study, we found that rhesus macaques who voluntarily drink $1.5 \mathrm{~g} / \mathrm{kg} /$ day ethanol for the first 60 days of gestation, which corresponds to the first trimester in human pregnancy, have perturbed placental perfusion at mid- and late gestation. Due to the fact that many pregnancies are unplanned or unrecognized until mid- to late-first trimester ${ }^{2-4}$, this NHP model is relevant for studying the outcomes of pregnancies at risk for fetal alcohol spectrum disorder due to alcohol consumption prior to pregnancy recognition. Compared with other animal models studying the effects of fetal alcohol exposure, the NHP is advantageous due to its similar physiology to humans ${ }^{19}$, including the rates of absorption and metabolism of ethanol ${ }^{19}$, the ability to train animals to voluntarily drink alcohol (hence avoiding confounds associated with inducing maternal stress) ${ }^{31}$, and similarities in fetal development, particularly with regard to gestational timing ${ }^{17-18}$. Significant findings of decreased fetal oxygen availability, weight, and abnormal brain development were observed in ethanolexposed pregnancies at mid-gestation. Our findings highlight the ability of DCE-MRI and $T_{2}^{*}$ analyses as in vivo imaging modalities that have the sensitivity to characterize reduced intervillous perfusion and oxygen exchange with the fetal vasculature in response to an adverse in utero environment.

This study utilized a cross-sectional experimental design to focus on outcomes at two gestational time points of G110 and G135; 50 and 75 days following the last day of access to ethanol respectively. Therefore, although ethanol has been shown to acutely reduce fetal and maternal blood flow to the placenta in humans and other animal models ${ }^{6-8}$, the alterations to placental function observed here more likely result from longer-term developmental 
consequences of ethanol exposure during the periconceptional period. Prior rodent studies have identified the influence of periconceptional ethanol exposure on subsequent trophoblast survival, gene expression, and zonal distribution at later gestational ages ${ }^{32,33}$. Notably, in rodents, ethanol exposure early in pregnancy is associated with reduced trophoblast invasion of the spiral arteries and altered placental vascular development associated with impaired fetal growth ${ }^{34-36}$. Similar to the observations made in rodents, there were multiple findings suggesting aberrant placental function including decreased fetal weight and placental volume blood flow, a semi-quantitative measure of blood flow through the umbilical vein. Notably, there were no detectable changes in the pulsatility indices of both the uterine and umbilical arteries, which is not surprising given significant increases in the umbilical artery pulsatility index are estimated to occur only after more than $60 \%$ of the placental terminal vascular branches are destroyed ${ }^{37}$. Reduced maternal blood flow to the intervillous space, revealed with DCE-MRI, and decreased placental oxygen reserve, revealed in the analysis of placental $T_{2}{ }^{*}$ values, indicate that maternal delivery of oxygen to the fetus was lower in ethanol-exposed animals than in controls at G110.

In addition to alterations to placental function and fetal growth at G110, fetal brain development was also affected by first trimester ethanol exposure. Specifically, ethanol exposed fetuses showed significantly smaller fetal biparietal diameter and brain weight, consistent with prior MRI studies in murine ${ }^{38}$ and rat ${ }^{39-40}$ models demonstrating structural brain abnormalities resulting from first trimester ethanol exposure and microcephaly observed with fetal alcohol syndrome in human neonates ${ }^{41}$. Given that recent neuroimaging studies have revealed alterations in normal brain development in the setting of abnormal fetal growth ${ }^{42-45}$, it is possible that aberrant placental perfusion resulting in decreased oxygen availability contributed to the reduced fetal brain size. Interestingly, at G135, fetal and placental outcomes are less severe. While the mechanism for this discrepancy is unknown, it is consistent with placental adaptive capabilities to transient environmental perturbations as has been observed in early acute injury to macaque placentas ${ }^{46}$ when compared with chronic environmental perturbations throughout pregnancy ${ }^{24,47}$. Although the placental weight in ethanol exposed pregnancies compared to controls is less at G110, at G135 the placental weight was similar between both groups suggesting that the placenta partly compensates by altering its growth trajectory to support fetal growth, albeit that fetal weights are not fully restored to control weights.

Evaluation of uterine hemodynamics by Doppler-US did not detect significant differences in vascular impedance of the uterine artery or the cQuta in G110 animals, yet reduced blood flow through spiral arteries was observed with DCE-MRI at this gestational age (Table 3). This is likely a consequence of the Doppler measures reflecting vascular function proximal to the placenta which limits its utility to assess the placental microcirculation as well as the limited field of view with Doppler-US measurements. In contrast, with MRI it is possible to map blood flow to the entire placenta concurrently, within a single field of view. The ability to characterize all spiral arteries on an individual level provides improved sensitivity over existing methods for in utero surveillance of placental perfusion. Thus, DCE-MRI is an imaging strategy that can enable spatial and quantitative characterization of maternal perfusion of the placental intervillous space and identify aberrant placental hemodynamics in pathologic conditions from environmental perturbations. 
A drawback of DCE-MRI as a tool for characterizing pregnancy in humans relates to the need for gadolinium-based contrast reagents. Such reagents are not typically administered to pregnant women due to potential risks to the fetus ${ }^{48,49}$. Recently, we demonstrated that $T_{2}{ }^{*}$ based MRI measurements can also be used to characterize the entire placenta at the level of individual spiral artery perfusion domains. Specifically, whereas DCE-MRI provides estimates of blood flow, $F$, through each spiral artery ${ }^{13}$, we have proposed an analysis of $T_{2}{ }^{*}$ parameter maps to estimate the flow-permeability ratio, $F\left({ }_{v} P S\right)$ for each spiral artery ${ }^{14}$. We observed that ethanol exposed animals which exhibited reduced placental blood flow at G110 by DCE-MRI also demonstrated reduced average $T_{2}{ }^{*}$ and corresponding $F(v P S)$. This pattern provides promising evidence that $T_{2}^{*}$ measurements can be used to assess placental function at the level of individual spiral arteries in human pregnancies, which is a focus of ongoing research initiatives funded by the Human Placenta Project.

In summary, in this study of voluntarily drinking rhesus macaques, first trimester ethanol exposure leads to impaired maternal placental perfusion at mid- to late-gestation, resulting in decreased oxygen availability, and negative effects on fetal brain development. As there is no cure for the adverse fetal affects from alcohol and it can result in lifelong implications, ideally it is prudent for women trying to conceive to avoid alcohol consumption. However, for unplanned pregnancies and those at risk of early prenatal alcohol exposure, the MRIbased measurements described here provide new potential non-invasive obstetric tools with increased sensitivity for characterizing placental health. These novel imaging strategies have translational potential as clinical methods for earlier identification of at-risk pregnancies to facilitate intervention and improve pregnancy outcomes prior to the onset of fetal and neonatal morbidity and mortality.

\section{Acknowledgments}

Funding: This study was supported by NIH/NIAAA grant R01 AA021981, R01 HD086331, P51-OD-011092, K12HD000849

\section{References}

1. Floyd RL, et al. Monitoring prenatal alcohol exposure. Am J Med Genet C Semin Med Genet. 2004; 27:3-9.

2. Edwards EM, Werler MM. Alcohol consumption and time to recognition of pregnancy. Matern Child Health J. 2006; 10(6):467-472. [PubMed: 16763772]

3. Floyd RL, Decoufle P, Hungerford DW. Alcohol use prior to pregnancy recognition. Am J Prev Med. 1999; 17(2):101-107. [PubMed: 10490051]

4. Alcohol and Pregnancy. Centers for Disease Control and Prevention; website: http://www.cdc.gov/ vitalsigns/fasd/. Updated February 2, 2016. Last accessed 11/13/16

5. Idanpaan-Heikkila $\mathbf{J}$, et al. Elimination and metabolic effects of ethanol in mother, fetus, and newborn infant. Am J Obstet Gynecol. 1972; 112:387-393. [PubMed: 5060384]

6 . Falconer J. The effect of maternal ethanol infusion on placental blood flow and fetal glucose metabolism in sheep. Alcohol Alcohol. 1990; 25(4):413-6. [PubMed: 2222575]

7. Kochunov P, Castro C, Davis D, Dudley D, Wey H, et al. Fetal brain during a binge drinking episode: A dynamic susceptibility contrast MRI fetal brain perfusion study. Neuroreport. 2010; 21(10):716-721. [PubMed: 20505549] 
8. Siler-Khodr TM, Yang Y, Grayson MH, Henderson GI, Lee M, et al. Effect of ethanol on thromboxane and prostacyclin production in the human placenta. Alcohol. 2000; 21(2):169-180. [PubMed: 10963940]

9. O'Leary CM, et al. The effect of maternal alcohol consumption on fetal growth. BJOG. 2009; $116(3)$

10. Hannigan JH, Armant DR. Alcohol in pregnancy and neonatal outcome. Semin Neo. 2000; 5(3): 243-54.

11. Taylor SM, Heron AE, Cannell GR, Florin TH. Pressor effect of ethanol in the isolated perfused human placental lobule. Eur J Pharmacol. 1994; 270:371-74. [PubMed: 7805788]

12. Guttmacher AE, Maddox YT, Spong CY. The human placenta project: placental structure, development, and function in real time. Placenta. 2014; 35(5):303-304. [PubMed: 24661567]

13. Frias AE, Schabel MC, Roberts V, Tudorica A, Grigsby PL, et al. Using dynamic contrast enhanced MRI to quantitatively characterize maternal vascular organization in the primate placenta. Magn Reson Med. 2015; 73(4):1570-1578. [PubMed: 24753177]

14. Schabel MC, Roberts VHJ, Lo JO, Platt S, Grant KA, et al. Functional imaging of the non-human primate placenta with endogenous blood oxygen level-dependent contrast. Magn Reson Med. 2016; 76(5):1551-1562. [PubMed: 26599502]

15. Oh KY, Roberts VH, Schabel MC, Grove KL, Woods M, Frias AE. Gadolinium chelate contrast material in pregnancy: Fetal biodistribution in the nonhuman primate. Radiology. 2015; 276(1): 110-118. [PubMed: 25763829]

16. Sorensen A, Peters D, Frund E, Lingman G, Christiansen O, Uldbjerg N. Changes in human placental oxygenation during maternal hyperoxia estimated by blood oxygen level-dependent magnetic resonance imaging (BOLD MRI). Ultrasound Obstet Gynecol. 2013; 42(3):310-314. [PubMed: 23303592]

17. Carter AM. Animal models of human placentation—a review. Placenta. 2007; 28(Suppl A):S41S47. [PubMed: 17196252]

18. Blankenship TN, Enders AC. Modification of uterine vasculature during pregnancy in macaques. Microsc Res Tech. 2003; 60(4):390-401. [PubMed: 12567396]

19. Green KL, Szeliga KT, Bowen CA, Kautz MA, Azarov AV, Grant KA. Comparison of ethanol metabolism in male and female cynomolgus macaques (Macaca fascicularis). Alcohol Clin Exp Res. 1999; 23(4):611-616. [PubMed: 10235296]

20. Grant KA, Leng X, Green HL, Szeliga KT, Rogers LS, Gonzales SW. Drinking typography established by scheduled induction predicts chronic heavy drinking in a monkey model of ethanol self-administration. Alcohol Clin Exp Res. 2008; 32:1824-38. [PubMed: 18702645]

21. Vivian JA, Green HL, Young JE, Majerksy LS, Thomas BW, Shively CA, Tobin JR, Nader MA, Grant KA. Induction and maintenance of ethanol self-administration in cynomolgus monkeys (Macaca fascicularis): long-term characterization of sex and individual differences. Alcohol Clin Exp Res. 2001; 25(8):1087-1097. [PubMed: 11505038]

22. Hougaard KS, Hansen AM. Enhancement of developmental toxicity effects of chemicals by gestational stress. A review. Neurotoxicol Teratol. 2007; 29(4):425-445. [PubMed: 17433620]

23. Falk JL. Production of polydipsia in normal rats by an intermittent food schedule. Science. 1961; 133(3447):195-196. [PubMed: 13698026]

24. Frias A, Morgan T, Evans A, Rasanen J, Oh K, Thornburg K, Grove K. Maternal high-fat diet disturbs uteroplacental hemodynamics and increases the frequency of stillbirth in a nonhuman primate model of excess nutrition. Endo. 2011; 152(6):2456-2464.

25. Konje JC, Kaufmann P, Bell SC, Taylor DJ. A longitudinal study of quantitative uterine blood flow with the use of color power angiography in appropriate for gestational age pregnancies. Am J Obstet Gynecol. 2001; 185(3):608-613. [PubMed: 11568786]

26. Acharya G, Sitras V, Erkinaro T, et al. Experimental validation of uterine artery volume blood flow measurements by Doppler ultrasonography in pregnant sheep. Ultrasound Obstet Gynecol. 2007; 29(4):401-406. [PubMed: 17390334]

27. Acharya G, Wilsgaard T, Berntsen GK, Maltau JM, Kiserud T. Doppler-derived umbilical artery absolute velocities and their relationship to fetoplacental volume blood flow: a longitudinal study. Ultrasound Obstet Gynecol. 2005; 25(5):444-453. [PubMed: 15816007] 
28. Wang X, Pettersson D, Studholme C, Kroenke C. Characterization of laminar zones in the midgestation primate brain with magnetic resonance imaging and histological methods. Front Neuroanat. 2015; 9:147. [PubMed: 26635541]

29. Schabel MC, Morrell GR. Uncertainty in T(1) mapping using the variable flip angle method with two flip angles. Phys Med Biol. 2009; 54(1):N1-8. [PubMed: 19060359]

30. Tarantal AF, Hendrickx AG. Prenatal growth in the cynomolgus and rhesus macaque (Macaca fascicularis and Macaca mulatta): a comparison by ultrasonography. Ameroican Journal of Primatology. 1988; 15(4):309-323.

31. Lan N, Chiu MP, Ellis L, Weinberg J. Prenatal alcohol exposure and prenatal stress differentially alter glucocorticoid signaling in the placenta and fetal brain. Neuroscience. 2015; (15):S03064522. 00790-3.

32. Gardebjer EM, Cuffe JSM, Pantaleon M, Wlodek ME, Moritz KM. Periconceptional alcohol consumption causes fetal growth restriction and increases glycogen accumulation in the late gestation rate placenta. Placenta. 2014; 35(1):50-57. [PubMed: 24239160]

33. Kalisch-Smith JI, Outhwaite JE, Simmons DG, Pantaelon M, Moritz KM. Alcohol exposure impairs trophoblast survival and alters subtype-specific gene expression in vivo. Placenta. 2016; 46:84-97.

34. Gundogan F, Gilligan J, Qi W, Chen E, Naram R, de la Monte SM. Dose effect of gestational ethanol exposure on placentation and fetal growth. Placenta. 2015; 36(5):523-30. [PubMed: 25745824]

35. Gundogan F, Elwood G, Longato L, Tong M, Feijoo A, et al. Impaired placentation in fetal alcohol syndrome. Placenta. 2008; 29(2):148-157. [PubMed: 18054075]

36. Gundogan F, Gilligan J, Ooi J, Sung J, Naram R, et al. Dual mechanisms of ethanol-impaired placentation: experimental model. J Clin Exp Pathol. 2013; 3(2)

37. Thompson RS, Trudinger BJ. Doppler waveform pulsatility index and resistance, pressure and flow in the umbilical placental circulation: an investigation using a mathematical model. Ultrasound Med Bio. 1990; 116(5):449-458.

38. Parnell SE, Holloway HE, Baker LK, Styner MA, Sulik KK. Dysmorphogenic effects of first trimester-equivalent ethanol exposure in mice: a magnetic resonance microscopy-based study. Alcohol Clin Exp Res. 2014; 38(7):20008-2014.

39. Leigland LA, Ford MM, Lerch JP, Kroenke CD. The influence of fetal ethanol exposure on subsequent development of the cerebral cortex as revealed by magnetic resonance imaging. Alcohol Clin Exp Res. 2013; 37(6):924-932. [PubMed: 23442156]

40. Leigland LA, Budde MD, Cornea A, Kroenke CD. Diffusion MRI of the developing cerebral cortical gray matter can be used to detect abnormalities in tissue microstructure associated with fetal ethanol exposure. Neuroimage. 2013; 83:1081-1087. [PubMed: 23921100]

41. American Academy of Pediatrics. Committee on substance abuse and committee on children with disabilities. Fetal alcohol syndrome and alcohol-related neurodevelopmental disorders. Pediatrics. 2000; 106(2Pt 1):358-361. [PubMed: 10920168]

42. Jarvis S, Glinianaia SV, Torrioli MG, Platt MJ, Miceli M, Jouk PS, et al. Cerebral palsy and intrauterine growth in single births: European collaborative study. Lancet. 2003; 362(9390):11061111. [PubMed: 14550698]

43. Bergvall N, Iliadou A, Johansson S, Tuvemo T, Cnattingius S. Risks for low intellectual performance related to being born small for gestational age are modified by gestational age. Pediatrics. 2006; 117(3):e460-467. [PubMed: 16510624]

44. Bernstein IM, Horbar JD, Badger GJ, Ohlsson A, Golan A. Morbidity and mortality among verylow-birth-weight neonates with intrauterine growth restriction. The Vermont Oxford Network. Am J Obstet Gynecol. 2000; 182(1 Pt 1):198-206. [PubMed: 10649179]

45. Levine TA, Grunau RE, McAuliffe FM, Pinnamaneni R, Foran A, Alderdice FA. Early childhood neurodevelopment after intrauterine growth restriction: a systematic review. Pediatrics. 2015; 135(1):126-141. [PubMed: 25548332]

46. Roberts VH, Rasanen JP, Novy MJ, Frias A, Louey S, et al. Restriction of placental vasculature in a non-human primate: a unique model to study placental plasticity. Placenta. 2012; 33(1):73-76. [PubMed: 22030304] 
47. Lo JA, Schabel M, Roberts V, Morgan T, Rasanen J, Kroenke C, Shoemaker S, Spindel E, Grove K, Frias A. Vitamin C supplementation ameliorates the adverse effects of nicotine on placental hemodynamics and histology in non-human primates. Am J Obstet Gynecol. 2015; 212(3):370e18. (Epub ahead of print). [PubMed: 25725660]

48. Sundgren PC, Leander P. Is administration of gadolinium-based contrast media to pregnant women and small children justified? J Magn Reson Imaging. 2011; 34(4):750-757. [PubMed: 21928308]

49. Ray JG, Vermeulen MJ, Bharatha A, Montanera WJ, Park AL. Association between MRI exposure during pregnancy and fetal and childhood outcomes. JAMA. 2016; 316(9):952-961. [PubMed: 27599330] 


\section{CONDENSATION}

Alcohol Exposure In the First Trimester Adversely Impacts Placental Perfusion and Fetal Oxygen Availability in the Non-human Primate 

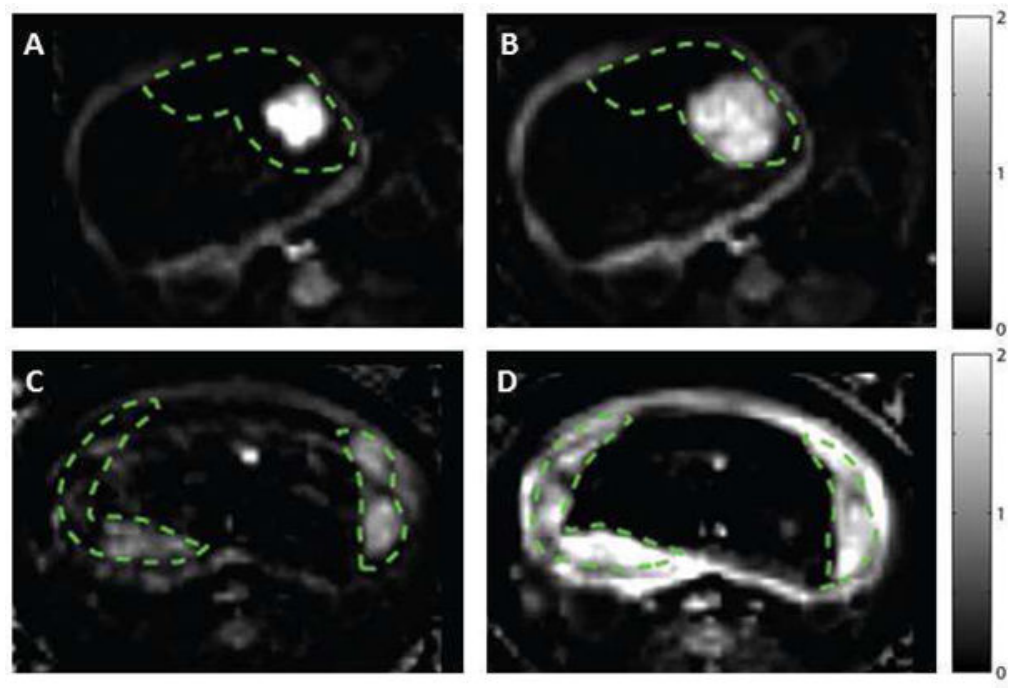

Figure 1. Placental contrast uptake at G135 in ethanol exposed (upper panels) vs. control (lower panels) animals at 30 seconds (left column) and 2.5 minutes (right column) after contrast agent injection

Relative tissue enhancement (as a fraction of baseline signal) is plotted at two time points for single axial slice through the uterus, with placenta delineated by the dashed green lines. In the EtOH exposed animal, there is avid enhancement near the spiral artery outlet (panel A), but a substantial portion of the placental tissue is not perfused, as indicated by the lack of late enhancement in panel B. In contrast, the control placenta enhances more gradually, with multiple sources visible in panel $\mathrm{C}$ and the placenta being nearly completely perfused at the second time point (panel D). 

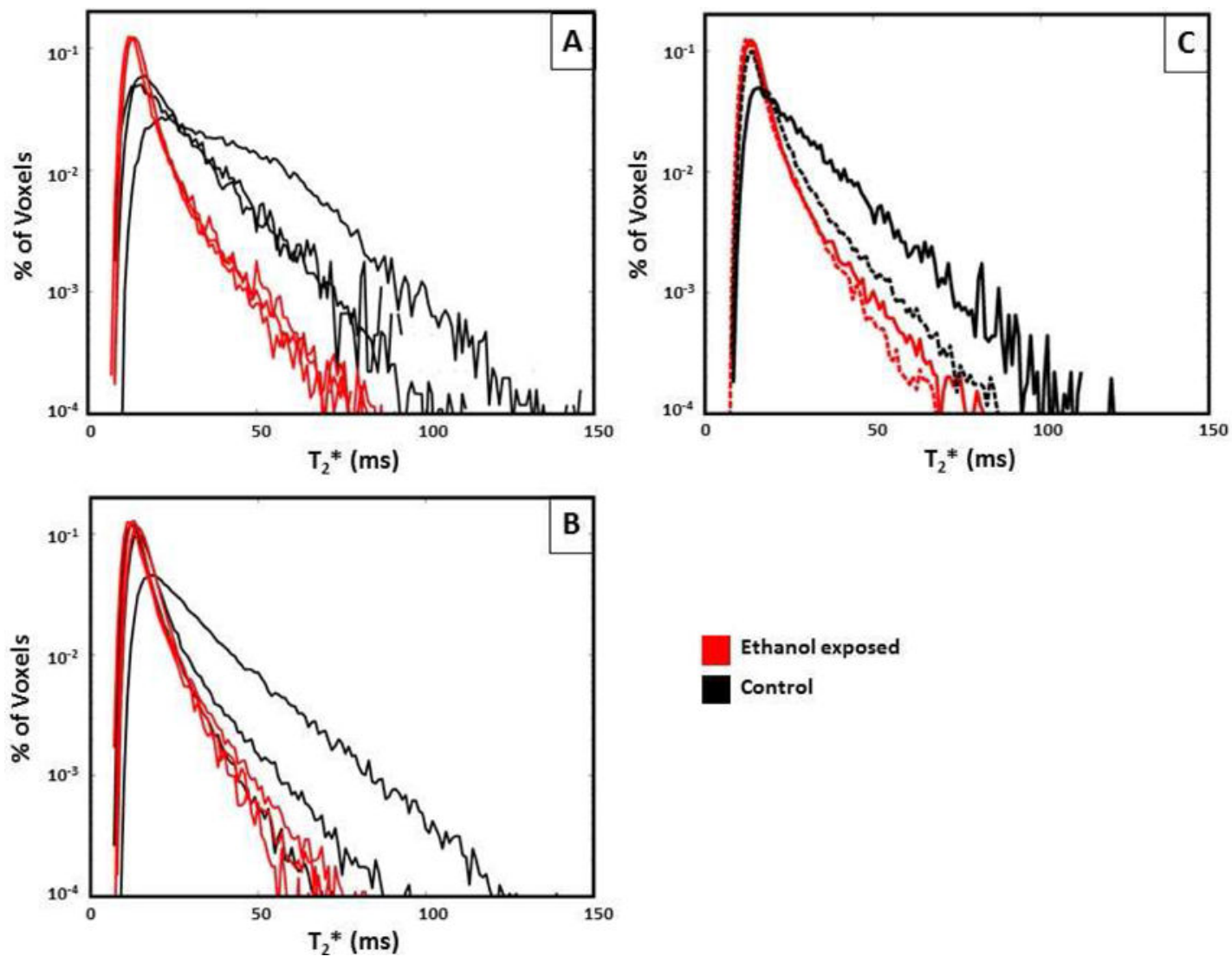

Ethanol exposed

Control

Figure 2. Histogram plots of $T_{2}^{*}$ versus percent of placental voxels displayed for ethanol exposed (red)_vs. control animals (black) at G110 (panel A), G135 (panel B), and median $\mathrm{T}_{2} *$ values (panel C) at both G110 (solid line) and G135 (dashed line)

Ethanol exposed animals had a significantly smaller fraction of large $\mathrm{T}_{2} *$ values compared to controls at G110 ( $\mathrm{p}=0.02)$ compared to G135 ( $\mathrm{p}=0.39)$, demonstrating decreased fetal oxygen availability in the former mid-gestation. 

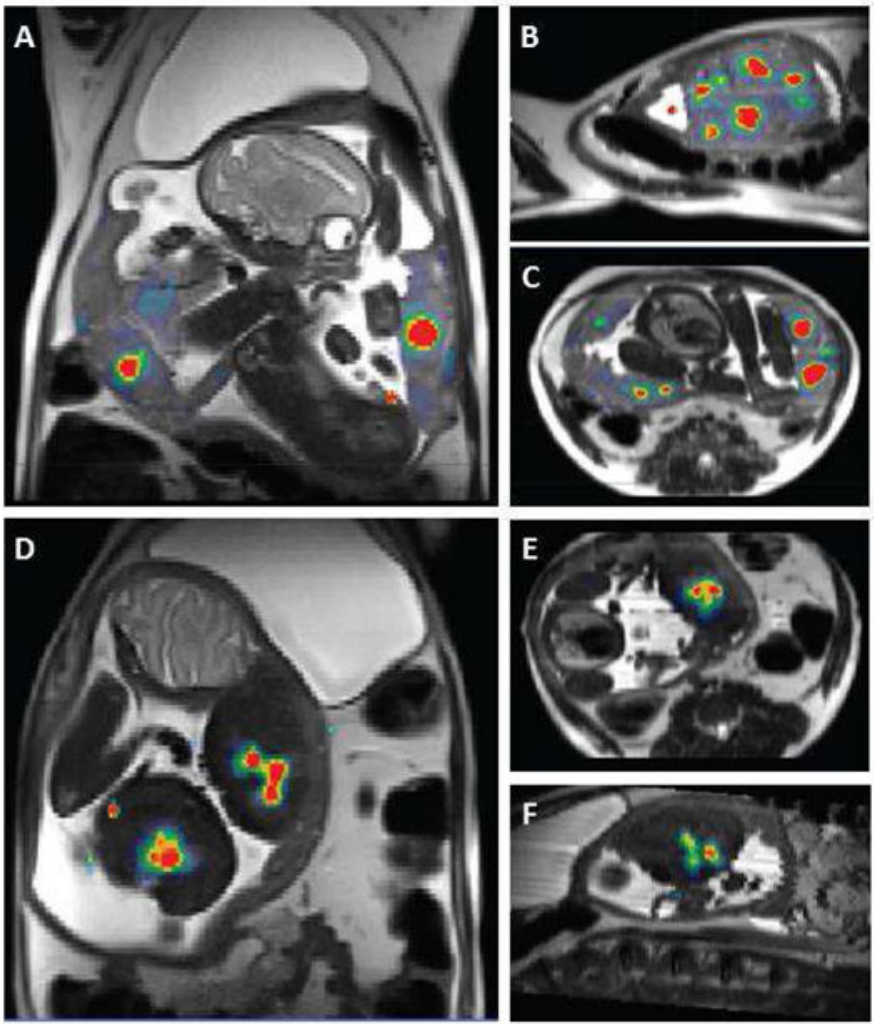

Figure 3. Placental blood flow from DCE-MRI superimposed on maps of $\boldsymbol{T}_{2} *$ values for a representative control (panels A-C) and EtOH exposed (panels D-F) animal shown in Figure 1 Blood flow values range from $0-2 \mathrm{ml} / \mathrm{ml} / \mathrm{min}$, and are plotted to emphasize the number and location of spiral artery outlets (red regions). $T_{2}{ }^{*}$ values are plotted on a linear grayscale from 0-120 milliseconds. In addition to larger number of spiral arteries seen in the control animal, its placenta shows uniformly higher $T_{2}{ }^{*}$ values relative to those in the EtOH exposed animal. 


\section{Table 1}

Fetal biometry

\begin{tabular}{lcccc}
\hline Parameter & \multicolumn{2}{c}{ Gestational day 110 } & \multicolumn{2}{c}{ Gestational day 135 } \\
& Control (n=3) & Ethanol (n=3) & Control (n=3) & Ethanol (n=3) \\
\hline BPD (mm) & $39 \pm 0.9$ & $35 \pm 1.1^{* *}$ & $45 \pm 0.6$ & $44 \pm 1.4$ \\
AC (cm) & $12 \pm 0.2$ & $11 \pm 0.8$ & $14 \pm 0.4$ & $13 \pm 1.9$ \\
FL (mm) & $29 \pm 0.4$ & $26 \pm 0.9^{*}$ & $37 \pm 1.9$ & $36 \pm 0.5$ \\
\hline
\end{tabular}

Definition of abbreviations:

$\mathrm{BPD}=$ biparietal diameter

Non-paired t-test used. Data are means \pm SD.

p $<0.05$,

*** $<<0.01$ 


\section{Table 2}

Maternal, fetal birth, and placental weights

\begin{tabular}{lcccc}
\hline Parameter & \multicolumn{2}{c}{ Gestational day 110 } & \multicolumn{2}{c}{ Gestational day 135 } \\
& Control (n=3) & Ethanol (n=3) & Control (n=3) & Ethanol (n=3) \\
\hline Maternal weight $(\mathrm{kg})$ & $7.5 \pm 1.7$ & $8.3 \pm 1.4$ & $7.5 \pm 0.9$ & $8.5 \pm 1.1$ \\
Fetal weight $(\mathrm{g})$ & $217 \pm 21$ & $175 \pm 8.4^{*}$ & $333 \pm 6.5$ & $318 \pm 3.6^{*}$ \\
Placental weight (g) & $75 \pm 17$ & $65 \pm 4.1$ & $78 \pm 2.3$ & $83 \pm 10.3$ \\
Fetal sex (male:female) & $1: 2$ & $0: 3$ & $1: 2$ & $1: 2$ \\
\hline
\end{tabular}

Non-paired t-test used. Data are means \pm SD.

p $<0.05$ 
Table 3

Doppler ultrasound and Dynamic contrast-enhanced MRI measurements of placental function and oxygenation

\begin{tabular}{|c|c|c|c|c|}
\hline \multirow[t]{2}{*}{ Parameter } & \multicolumn{2}{|c|}{ Gestational day 110} & \multicolumn{2}{|c|}{ Gestational day 135} \\
\hline & Control $(n=3)$ & Ethanol(n=3) & Control $(n=3)$ & Ethanol(n=3) \\
\hline Uterine artery PI & $0.65 \pm 0.1$ & $0.82 \pm 0.1$ & $0.86 \pm 0.3$ & $0.8 \pm 0.01$ \\
\hline Umbilical artery PI & $1.22 \pm 0.1$ & $1.7 \pm 0.3$ & $1.15 \pm 0.1$ & $1.35 \pm 0.5$ \\
\hline cQuta (ml/min/kg) & $48 \pm 23$ & $19 \pm 11$ & $34 \pm 22$ & $27 \pm 0.1$ \\
\hline cQuv & $22 \pm 4.8$ & $15 \pm 0.7^{*}$ & $29 \pm 5.9$ & $26 \pm 18$ \\
\hline Total Placental blood flow $(\mathrm{ml} / \mathrm{min})^{\xi}$ & $681 \pm 40$ & $284 \pm 142^{* * *}$ & $558 \pm 189$ & $501 \pm 166$ \\
\hline Median $F(v P S)\left(\mathrm{cm}^{3}\right)$ & $(4.4 \pm 0.5) \times 10^{-6}$ & $(0.69 \pm 0.08) \times 10^{-6}$ & $(1.7 \pm 0.2) \times 10^{-6}$ & $(1.0 \pm 0.4) \times 10^{-6}$ \\
\hline \multicolumn{5}{|l|}{ Definition of abbreviations: } \\
\hline \multicolumn{5}{|l|}{$\xi_{=}$obtained by DCE-MRI } \\
\hline \multicolumn{5}{|c|}{$\mathrm{PI}=$ pulsatility index, $\mathrm{VTI}=$ velocity time integral } \\
\hline \multicolumn{5}{|c|}{ CSA $($ cross section of uterine artery $)=\pi(\text { diameter } / 2)^{2}$} \\
\hline \multicolumn{5}{|c|}{ Vmean $($ mean velocity $)=0.5 \times$ maximum umbilical vein velocity } \\
\hline \multicolumn{5}{|c|}{ cQuta (uterine artery blood flow) $=$ VTI $\times$ CSA $\times$ HR adjusted for maternal weight } \\
\hline \multicolumn{5}{|c|}{ cQuv (placental volume blood flow $)=$ Vmean $\times$ CSA $\times 60$} \\
\hline \multicolumn{5}{|l|}{$F=$ maternal volume blood flow } \\
\hline \multicolumn{5}{|l|}{$V=$ volume fraction of the intervillous space } \\
\hline \multicolumn{5}{|l|}{$P S=$ oxygen permeability surface area } \\
\hline \multicolumn{5}{|l|}{$F /(v P S)=$ flow permeability ratio } \\
\hline \multicolumn{5}{|c|}{ Non-paired t-test used. Data are means $\pm \mathrm{SD}$. } \\
\hline$*$ p $<0.05$ & & & & \\
\hline *** $<<0.01$ & & & & \\
\hline
\end{tabular}


Table 4

Brain measurements obtained by MRI

\begin{tabular}{lcccc}
\hline Parameter & \multicolumn{2}{c}{ Gestational day 110 } & \multicolumn{2}{c}{ Gestational day 135 } \\
& Control $(\mathbf{n = 3})$ & Ethanol $(\mathbf{n = 3})$ & Control $(\mathbf{n = 3})$ & Ethanol $(\mathbf{n = 3})$ \\
\hline Brain weight $(\mathrm{g})$ & $13 \pm 1.1$ & $11 \pm 0.9^{*}$ & $20 \pm 0.6$ & $19 \pm 0.7$ \\
Brain volume $\left(\mathrm{mm}^{3}\right)$ & $20097 \pm 2214$ & $17066 \pm 1090$ & $31003 \pm 914$ & $29173 \pm 3003$ \\
Brain surface area $\left(\mathrm{mm}^{2}\right)$ & $6133 \pm 527$ & $5372 \pm 499$ & $11530 \pm 443$ & $10838 \pm 497$ \\
\hline & & & \\
Non-paired t-test used. Data are means $\pm \mathrm{SD}$. & & & \\
$*$ & & &
\end{tabular}

p $<0.05$ 